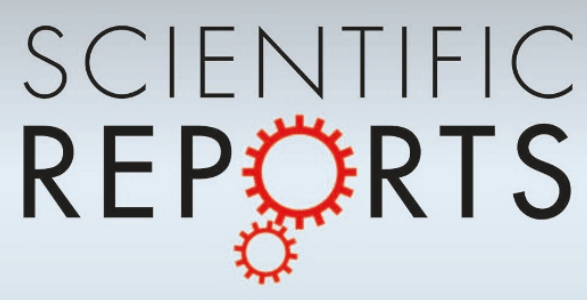

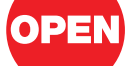

SUBJECT AREAS:

SYNTHESIS AND

PROCESSING

MATERIALS FOR ENERGY AND

CATALYSIS

OPTICAL MATERIALS

BATTERIES

Received

1 November 2012

Accepted

23 November 2012

Published

10 December 2012

Correspondence and requests for materials should be addressed to J.K. (jaekook@ chonnam.ac.kr)

* These authors contributed equally to this work.

\section{Pyro-Synthesis of Functional Nanocrystals}

\author{
Jihyeon Gim*, Vinod Mathew'* , Jinsub Lim', Jinju Song', Sora Baek', Jungwon Kang' , Docheon Ahn², \\ Sun-Ju Song', Hyeonseok Yoon ${ }^{3} \&$ Jaekook Kim
}

'Department of Materials Science and Engineering, Chonnam National University, 300 Yongbongdong, Bukgu, Gwangju 500757, South Korea, ${ }^{2}$ Beamline Research Division, Pohang Accelerator Laboratory, Pohang 790-784, South Korea, ${ }^{3}$ Department of Polymer and Fiber System Engineering, Chonnam National University, 300 Yongbongdong, Bukgu, Gwangiu 500-757, South Korea.

Despite nanomaterials with unique properties playing a vital role in scientific and technological advancements of various fields including chemical and electrochemical applications, the scope for exploration of nano-scale applications is still wide open. The intimate correlation between material properties and synthesis in combination with the urgency to enhance the empirical understanding of nanomaterials demand the evolution of new strategies to promising materials. Herein we introduce a rapid pyro-synthesis that produces highly crystalline functional nanomaterials under reaction times of a few seconds in open-air conditions. The versatile technique may facilitate the development of a variety of nanomaterials and, in particular, carbon-coated metal phosphates with appreciable physico-chemical properties benefiting energy storage applications. The present strategy may present opportunities to develop "design rules" not only to produce nanomaterials for various applications but also to realize cost-effective and simple nanomaterial production beyond lab-scale limitations.

rospective advancements in next-generation technologies are largely dictated by material properties. Owing to their confined particle-size dimensions, nanomaterials offer tremendous opportunities to realize exceptional physico-chemical properties in existing or new compounds ${ }^{1,2}$. Consequently, the generation and/or customization of functional nanocrystals by different synthetic strategies have become hugely significant. Low temperature syntheses present an efficient approach to produce innovative nanomaterials with exceptional performances suited to various disciplines including optical, electrical, magnetic, sensing, catalyst, targeted drug carrier and biomedical applications $\mathrm{s}^{3,4}$. One of the classic examples in the field of energy storage is the case of olivine-structured $\mathrm{LiFePO}_{4}$. The poorly conducting material that was initially introduced as a cathode demonstrating nominal electrochemical properties has now emerged as a prominent high-performance Li-ion battery electrode with outstanding performance by adopting technologies of nano-sizing $(<100 \mathrm{~nm})^{5-7}$, morphology tailoring ${ }^{8,9}$, conductive-phase inclusions ${ }^{10,11}$, aliovalent substitution ${ }^{12}$ and nanostructuring ${ }^{13,14}$. Nonetheless, the efficacy of these customized technologies in attaining an optimized correlation between efficient synthesis, competence and commercial viability still remains a stiff challenge.

The urge for targeted nanomaterial synthesis sparked off developments in specialized techniques involving solution or gas phases ${ }^{15,16}$. Despite this, conventional solid-state methods have remained as the preferred choice for large-scale industrialization. Notwithstanding the limitations and benefits exclusive to the synthesis adopted, several techniques including solid-state, sol-gel, hydrothermal, solvothermal, mechanochemical, electrochemical, and gas-phase microfluidic methods have been successful in furthering the empirical understanding on functional nanomaterials ${ }^{17-28}$. However, a majority of these methods, including the recent microwave ${ }^{29}$ and combustion $^{30}$ approaches risk shortcomings of complex procedures, time-consuming separation techniques, detrimental particle growth, economic viability, and commercial feasibility. Further, undesirable outcomes such as sample impurities and/or expensive procedures ultimately make such lab-scale synthesis less attractive. Hence, creative strategies involving versatile, straightforward, efficient, rapid, and timely synthesis from the perspective of simple large-scale industrialization is worth pursuing; though undertaking such an effort is no trivial task. Utilizing the polyol method, which usually involves the reaction of metallic precursors in a polyol-based environment maintained near or at its boiling point, to synthesize highly crystalline nanoparticles could appear very promising provided the lengthy/complicated reaction procedures were eliminated ${ }^{31,32}$. The polyol-assisted pyro-synthesis introduced here generates functional nanocrystals in open-air conditions under very short reaction times. Since 
polyol costs are comparable or lower than that of the commonly used solvents for wet chemical synthesis, the present work may provide orientation towards visualizing cost-effective and simple strategies of nanomaterial production beyond laboratory-scale limitations.

\section{Results}

Materials synthesis. The pyro-synthesis introduced herein presents a straightforward approach combining three strategies; first, to exploit the feasibility of utilizing a polyol-medium to produce highly crystalline nanoparticles; second, to ensure sustained combustion by using a highly combustible and low-cost fuel; and third, to minimize heat dissipation by maintaining the polyol-fuel solution at or near the fuel combustion temperature prior to combustion. As shown in Fig. 1, the polyol-assisted rapid pyro-synthesis procedure consists of two simple stages. The initial stage involves the preparation of a precursor solution by dissolving the metal salts $\left(\mathrm{M}^{\mathrm{n}+}\right)$ in a polyol (polyhydric/unsaturated aliphatic/alicyclic) medium. In the second stage, the flammable precursor solution is ignited with a torch that results in a rapid precipitation of highly crystalline nanoparticles. The polyol here acts as a primary fuel to induce a flame which can instantly provide ultrahigh thermal energy to surroundings. While the polyol undergoes fast combustion (exother$\mathrm{mic}$ ), the precursors thermochemically decompose (endothermic) and nucleate under oxygen-limited atmosphere by useful consumption of the thermal energy released during the exothermic reaction. The entire process occurs dynamically within a few seconds. The high energy generated and short reaction time facilitate rapid nucleation and suppress grain growth, which can in turn lead to the formation of highly crystalline ultrafine particles.

Specifically, the pyro-synthesis of olivine-type $\mathrm{LiFePO}_{4}$ nanoparticles employs lithium acetate hydrate, metal acetate, and phosphoric acid as the starting materials. During olivine synthesis by pyro-technique, in addition to the aforementioned mechanism, a co-operative interaction between polyol and phosphoric acid further contributes to the formation of nanoparticles. Under the polyol-rich condition, a part of the polyol can be pyrolyzed to carbonized structures although most undergo combustion to supply the energy for nanoparticle formation. Interestingly, the presence of phosphoric acid accelerates the carbonization of polyol. Precisely, phosphoric acid catalyzes the dehydration reaction of the polyol to yield carbocations and carboncarbon double bonds, which results in the generation of carbonized structures at high temperatures ${ }^{33}$. The carbonized structures act as physical barriers to prevent particle growth at elevated flame (or oxidation reaction) temperatures. Consequently, the $\mathrm{LiFePO}_{4}$ nanoparticles are coated with a thin, conductive carbon layer, which can prove to be advantageous for electrochemical applications. Unlike the characteristic dark gray color of $\mathrm{LiFePO}_{4}$, the carbon-encapsulated pyro-olivine powder is black in color (see Supplementary Fig. S1 online). The color variation clearly suggests that carbon-coated olivine nanoparticles are produced during pyro-synthesis and support the aforementioned discussion on the role of phosphoric acid in carbon coating. The presence of carbon coating has also been confirmed by thermal, elemental and high resolution transmission electron microscopy (HRTEM) studies discussed later.

Besides $\mathrm{LiFePO}_{4}$, we used the polyol-assisted pyro-synthetic approach to prepare pure metal oxides, sulfides and noble metals such as $\mathrm{ZnO}$ and $\mathrm{TiO}_{2}, \mathrm{CdS}$, and $\mathrm{Ag}$, useful for field emission display, optical, photovoltaic and biological applications respectively ${ }^{34-38}$. Unlike the case of $\mathrm{LiFePO}_{4}$, the characteristic yellow-orange color indirectly ascertains the absence of carbon in CdS (see Supplementary Fig. S1 online). The observation was further confirmed using HRTEM and is discussed later.

Thermodynamic considerations. The thermodynamic role of the polyol (polyhydric alcohol fuel) must be understood in terms of reaction catalyst for a given thermochemical reaction process. Model reactions for the binary compounds, $\mathrm{ZnO}$ and $\mathrm{TiO}_{2}$ were studied to describe the influence of polyol on the pyro-synthetic reaction. The reversible transformation reaction of the multiphase chemical system at isobaric condition results in spontaneous exothermic reaction with more negative values in the Gibbs free energy change $(\Delta G)$ for the hydrocarbon-based polyol-assisted reaction compared with that in no-hydrocarbon added classical methods over the wide range of temperatures (see Supplementary Fig. S2 online). The apparently lower $\Delta \mathrm{G}$ values lead one to conclude that the rapid pyro-synthesis appears to be a more energetically favorable process, yielding highly crystalline nanoparticles. It is fair to say that the rapid pyro-synthesis demonstrated in this study is a

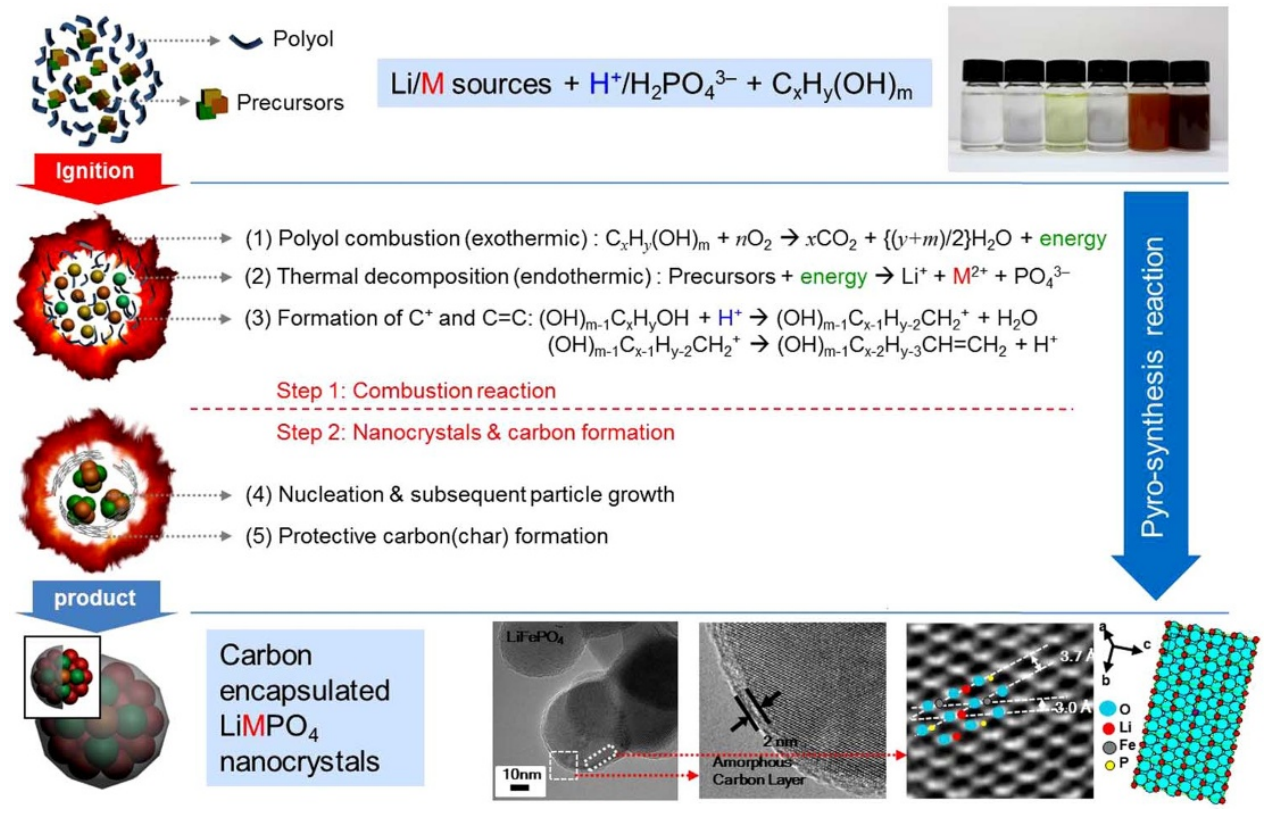

Figure 1 Rapid pyro-synthesis mechanism that explains typical production of carbon-encapsulated nanoparticles. The combustion of polyol with oxygen provides thermochemical energy for the pyrolytic decomposition of the precursors to finally yield nanoparticles under oxygen-limited condition, during which the rate of pyrolysis is much faster than the diffusion of oxygen. 
considerably simple process that may be considered for facile nanomaterials production. The associated chemical reaction equations at thermodynamic equilibrium and the calculated numerical thermodynamic quantities of each reaction as a function of temperature are provided in Supplementary Tables S1 to S5 online.

Structural and microscopic characterizations. X-ray diffraction (XRD) patterns reveal reflection lines corresponding to the anticipated pure single phases (Fig. 2). The crystal systems were modeled as a hexagonal for CdS (space group: $P 6_{3} m c$ ) and $\mathrm{ZnO}$ (space group: $\mathrm{P}_{3} m c$ ), tetragonal or anatase phase for $\mathrm{TiO}_{2}$ (space group: I41/amd), and orthorhombic phase for $\mathrm{LiFePO}_{4}$ (space group: Pnma), respectively. The absence of impurities in all the recorded patterns indicates the formation of pure crystalline phases under rapid reaction times. The refined lattice parameter values were obtained by fitting the XRD data using a whole-pattern profile matching method. The primary particle sizes, $d$, calculated from the X-ray line width using the Scherrer formula, range between 5 and $40 \mathrm{~nm}$ (see Supplementary Table S6 online). The lattice parameter values of $\mathrm{LiFePO}_{4}$ are slightly lower than those reported most probably due to its nanocrystalline characteristics ${ }^{39}$. The stoichiometric compositions of elements in $\mathrm{LiFePO}_{4}$ and CdS were also confirmed by chemical analysis (see Supplementary Table S7 online). The core-level spectra recorded for the prepared samples using X-ray photoelectron spectroscopy (XPS) established the oxidation states of constituent elements (see Supplementary Fig. S3 online). The field emission scanning electron microscopy (FE-SEM) images (Fig. 3, left) of the prepared samples indicate clearly that the resulting spherical nanoparticles did exhibit slight interparticle aggregation although their dispersion status somewhat depended a

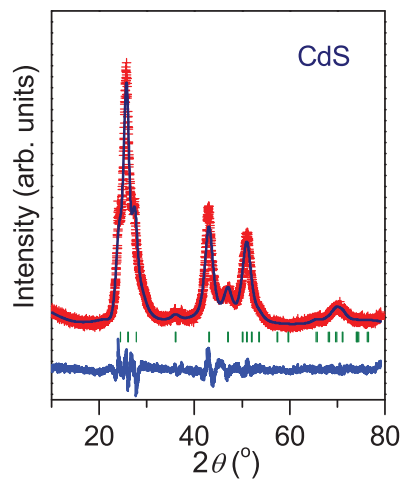

C

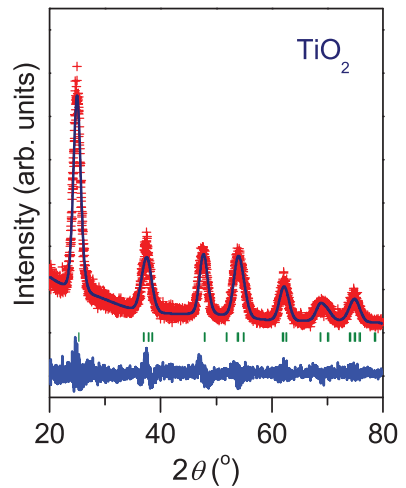

b

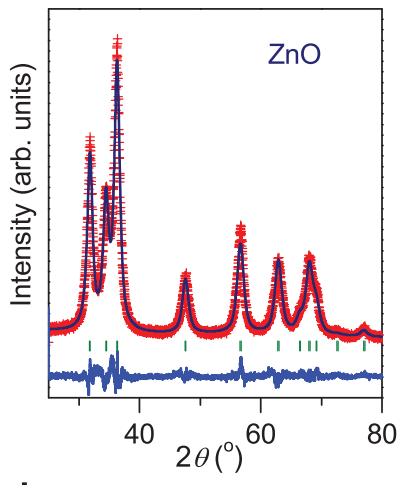

d

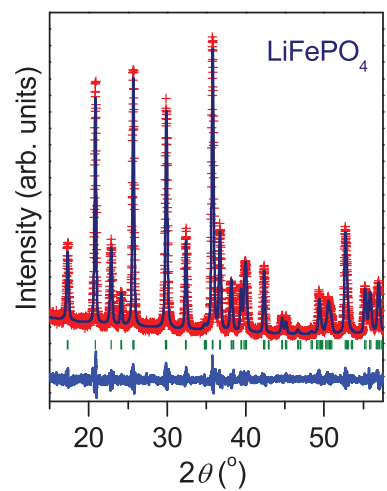

Figure $2 \mid$ Powder X-ray diffraction patterns of the samples prepared by rapid pyro-synthesis. The observed patterns (red dots) were fitted using a whole-pattern profile matching method. The calculated pattern (black line), the Bragg positions (green markers), and the difference curves (blue line) are provided for (a) CdS, (b) $\mathrm{ZnO}$, (c) $\mathrm{TiO}_{2}$ and (d) $\mathrm{LiFePO}_{4}$. on the type of precursor employed. More specifically, the SEM image of CdS shows apparently denser particle aggregation and that further re-dispersion efforts may be desirable. Transmission electron microscopy (TEM) observation was also performed to provide more insight into the structural and morphological sample characteristics (Fig. 3, center). The average particle-sizes of the samples ranging from a few to a few tens of nanometers correlates with the crystallite-sizes obtained from XRD studies (see Supplemetary Table S6 online). If the pyrolytic decomposition of the precursors proceed slowly under static conditions, the dense growth of grains may coalesce to yield apparently larger particles. However, under the present experimental conditions, the gaseous combustion products of the polyol prevents dense nucleation and simultaneously, the short overall reaction time prohibits coalescent grain growth leading to the formation of crystalline nanoparticles. In addition, the selected area electron diffraction (SAED) patterns (insets in Fig. 3, center) inform us that the $\mathrm{LiFePO}_{4}$ species is single-crystalline whereas $\mathrm{CdS}, \mathrm{ZnO}$, and $\mathrm{TiO}_{2}$ are polycrystalline. The high-resolution TEM images directly visualize the lattice fringes in the nanoparticles and confirm their crystalline nature (Fig. 3, right). The highly nanocrystalline characteristics confirm that the instantaneous thermal energy of the flame is sufficient enough for nucleation and subsequent crystal growth. Additional HRTEM images procured for $\mathrm{LiFePO}_{4}$ reveal that the nanoparticles are coated with a thin carbon layer of thickness $\sim 2 \mathrm{~nm}$ (see Supplementary Fig. S4 online). The carbon content was estimated to be approximately $5 \mathrm{wt} \%$ by elemental and thermal analyses (see Supplementary Table S8 and Fig. S5 online). Carbon coatings are generally pursued to scale-up electronic conductivities in characteristically poorly conducting nanomaterials and to prevent particle coalescence. Specific to energy storage applications, conductive coatings on electrode materials improve their electrochemical properties and performances. The nano-sized carbon coatings tend to permit unhindered ion-diffusion and in the case of $\mathrm{LiFePO}_{4}$

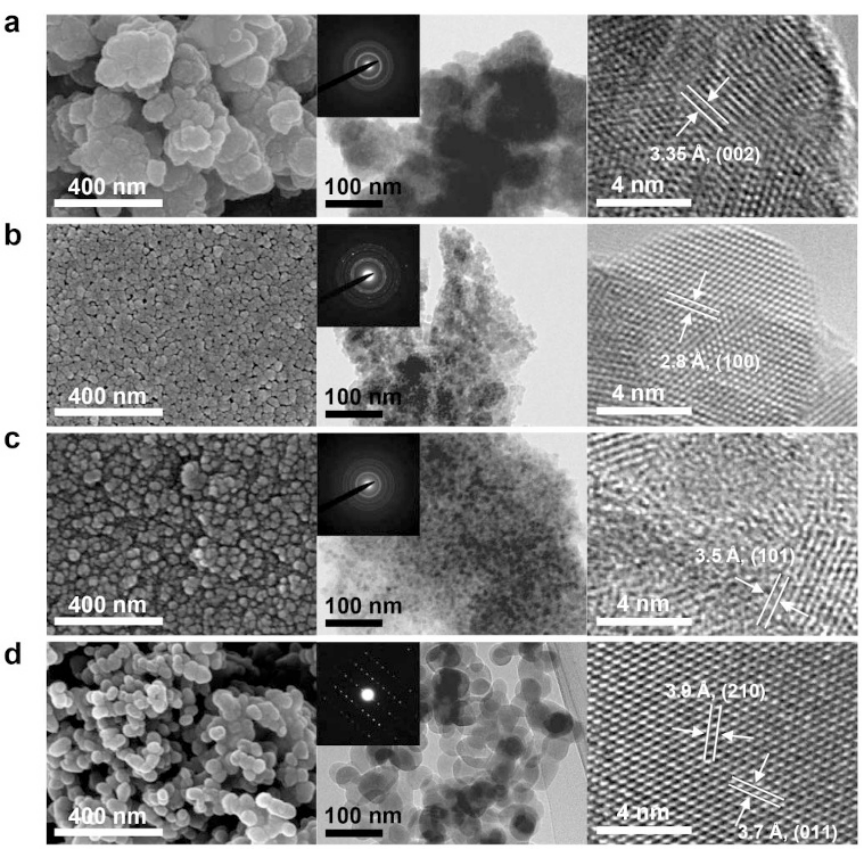

Figure 3 SEM/TEM images, HR-TEM images, and selected area electron diffraction (SAED) patterns of functional nanocrystals. The SEM and TEM images of (a) CdS, (b) $\mathrm{ZnO}$, (c) $\mathrm{TiO}_{2}$, and (d) $\mathrm{LiFePO}_{4}$ reveal wellordered primary particles with highly crystalline character and of diverse particle-sizes. SAED patterns (insets) indicate the poly-crystalline nature for $\mathrm{CdS}, \mathrm{ZnO}$, and $\mathrm{TiO}_{2}$, while that of olivine $\mathrm{LiFePO}_{4}$ reveals a clearly single-crystalline character. HR-TEM images visualizing the lattice fringes. 
synthesis, carbon can act as a reducing agent and prevent the otherwise easily-formed $\mathrm{Fe}^{3+}$ impurity. Hence, the rapid pyrosynthesis can serve as a useful strategy not only to realize particle minimization but also to obtain surface carbon coatings of energy storage nanomaterials. Given the fact that a starting precursor like phosphoric acid can accelerate polyol carbonization, the $\mathrm{LiFePO}_{4} / \mathrm{C}$ cathode produced by the rapid pyro-synthesis demonstrates impressive electrochemical abilities even under high charge/ discharge rates, the details of which are discussed in the following section. Further, the carbon coatings on $\mathrm{LiFePO}_{4}$ may be eliminated provided post-heat treatments are followed and pure olivine for other applications can be visualized. However, the high resolution TEM images of CdS reveal that bordering layers on the particle surfaces are non-existent unlike the coating observed for $\mathrm{LiFePO}_{4}$ particles. The finding thus strengthens the notion that carbon nanocoating in pyro-synthesis is largely dependent on precursors used (see Supplementary Fig. S4 online).

Optical and electrochemical properties. To demonstrate the dependability of producing nanomaterials by rapid pyro synthesis, the nanocrystalline samples were tested for material properties suited to various applications. The optical absorption properties of $\mathrm{CdS}$ and $\mathrm{ZnO}$ nanocrystals were analyzed by UV-vis absorption studies and its band gap energies $\left(E_{g}\right)$ were determined. The band gap energy $\left(E_{g}\right)$ values for $\mathrm{CdS}(2.13 \mathrm{eV})$ and $\mathrm{ZnO}(3.0 \mathrm{eV})$, obtained from the absorption curve $(\alpha h v)^{2}$ plots (see Supplementary Fig. S6 online), is slightly lower than the characteristic values of $\sim 2.4$ and $3.37 \mathrm{eV}$, respectively. The reduction in the band gap energies of semiconductors as $\mathrm{ZnO}$ and $\mathrm{CdS}$ is essential to realize the efficient use of sunlight by apparently more absorption of light in the visible region. Precisely, the UV-visible absorption spectra also indicates increased background in the visible light region $(>550 \mathrm{~nm})$ for $\mathrm{CdS}$ nanocrystals and an enhanced red-shift $(\geq 420 \mathrm{~nm}$ ) for $\mathrm{ZnO}$ (see Supplementary Fig. S6 online). The reduced band-gap energies and the enhanced UV-visible spectra contribute to enhanced photocurrent and high photo-catalytic efficiencies ${ }^{40,41}$ even though the CdS sample, in particular, revealed particle aggregation and further re-dispersion efforts may be desirable. The electrochemical properties of the $\mathrm{TiO}_{2}$ and $\mathrm{LiFePO}_{4}$ nanocrystals were measured by using them as anode and cathode of separate lithium test cells, respectively. Since the pyro-synthesis of $\mathrm{TiO}_{2}$ involved the use of carbon-based alkoxide as starting material, the as-prepared product required mild heating before performing electrochemical measurements. The XRD data confirmed the phase-purity of anatase $\mathrm{TiO}_{2}$ at $500^{\circ} \mathrm{C}$ (see Supplementary Fig. S7 online). An initial discharge capacity of $282 \mathrm{mAhg}^{-1}$, which corresponds to $85 \%$ utilization of theoretical capacities $\left(335 \mathrm{mAhg}^{-1}\right)$, is registered at $0.05 \mathrm{C}$ rate with steady capacity retentions ( $72 \%$ of initial capacity) for 50 cycles and impressive rate capabilities. Discharge capacities of $130 \mathrm{mAhg}^{-1}$ are delivered at $3.2 \mathrm{C}$ rates, which is $65 \%$ more than that observed for commercial anatase $\mathrm{TiO}_{2}$ anode (Aldrich) (see Supplementary Fig. S8 and detailed discussion online). The case of carbon-coated $\mathrm{LiFePO}_{4}$ has been investigated in detail due to its identification as a prominent cathode for high power $\mathrm{Li}$-ion batteries. The $\mathrm{LiFePO}_{4} / \mathrm{C}$ electrode delivers an initial capacity of $162 \mathrm{mAhg}^{-1}$, which corresponds to $95 \%$ theoretical capacity utilization, at $0.1 \mathrm{C}$ rate with consistent capacities up to 50 cycles and competent rate capabilities (see Supplementary Fig. S9 online). The electrochemical performance of the $\mathrm{LiFePO}_{4} / \mathrm{C}$ electrode well exceeds that exhibited by conventional solid-state $\mathrm{LiFePO}_{4}$ electrodes and the details are presented in the Supplementary Information. The enhanced specific capacities, cycle performance and competent rate capabilities compared to those of commercial/conventional electrodes may be attributed to the nano-sized particle characteristics (see Supplementary Figs. S10 and S11 online) with sufficient crystallinity and enhanced lithium ion diffusion ${ }^{42}$. Further, the presence of carbon facilitates improved electronic conductivity and enhanced electrochemical performance in pyro- $\mathrm{LiFePO}_{4}$.

We also extended the rapid pyro-synthesis to produce silver (Ag) nanoparticles due to its significance in various applications ${ }^{43,44}$. Although the polyol synthesis of Ag particles is known, the production of nanoparticles under very low reaction times remains important in this era of fast industrialization ${ }^{44,45}$. The prepared Ag powder displays its characteristic metallic color suggesting that carbon coating is indeed dependent on precursor choice and such a pyrolysis reaction appears to be thermodynamically more favorable than commercial methods (see Supplementary Fig. S12 and Tables S9 and S10 online). The XRD and XPS studies confirmed the formation of Ag metal. The average crystallite size was calculated to be $35 \mathrm{~nm}$ (see Supplementary Fig. S12 and Table S11 online). SEM and TEM images show agglomerated particles with diameters ranging from a few tens to a few hundreds of nanometers and distinguishable lattice fringes are visible (see Supplementary Fig. S13 online). Similar to the case of reducing bulk band gap energies to improve photo-catalytic efficiencies in $\mathrm{CdS}$ and $\mathrm{ZnO}$, the development of magnetic materials with tailored magneto-transport properties may also be feasible.

\section{Discussion}

The polyol synthesis of highly crystalline nanomaterials with promising physico-chemical properties is already known ${ }^{46-48}$. A majority of these reports indicate that precursor solutions are maintained close to the polyol boiling point for reaction durations of $\geq 1 \mathrm{~h}$ to facilitate nanocrystal formation and particle growth. The pyro-synthetic process introduces the possibility of producing nanomaterials in a very short reaction time of a few seconds under open-air environments. The energy produced during polyol combustion facilitates the production of highly crystalline nanomaterials and thereby ensures that energy consumption is maintained at a minimum. The one-step pyro-synthetic strategy useful to prepare carboncoated $\mathrm{LiFePO}_{4}$, a prospective cathode for high power Li-ion batteries, appears to overlook the requirements of repeated grinding/ pelleting procedures and inert environments usually required during commercial preparation of $\mathrm{LiFePO}_{4}{ }^{49-51}$. Further, the pyro-synthesis adopts a continuous sample processing technique in contradiction to the challenging batch-to-batch processing followed for commercial solid-state or sol-gel reactions to obtain $\mathrm{LiFePO}_{4}$. Therefore, the synthesis introduced here may provide solutions aimed at simplifying the process for nanomaterial production beyond laboratory scale limitations.

In summary, a rapid pro-synthetic strategy performed at very short reaction durations in open-air conditions to synthesize highly crystalline nanomaterials for useful battery, optical and bio-medical applications has been presented. The prepared nanomaterials of $\mathrm{CdS}$, $\mathrm{ZnO}, \mathrm{TiO}_{2}$ and $\mathrm{LiFePO}_{4}$ demonstrated decent optical and electrochemical properties though somewhat intense aggregation was visible for CdS. It may be possible to extend this strategy to develop various other metals, metal sulfides, metal oxides and phosphates for magnetic, electronic, ionic and metallic applications.

In the context of realizing nanomaterial production beyond labscale limitations, it is essential to evolve simple and cost-effective strategies. The pyro-synthetic process may offer opportunities to scale-up production of nanomaterials like $\mathrm{LiFePO}_{4}$ from the perspective of cost and simplification. Polyol costs are substantially lower, if not comparable, than that of common solvents used in wet chemical syntheses of nanomaterials (see Supplementary Table S12 and discussion online). More importantly, $\mathrm{LiFePO}_{4}$ is produced directly by a continuous processing technique that is relatively simpler than the batch-by-batch processing in commercial techniques (see Supplementary Fig. S14 and related discussion online). Therefore, the current pyro-synthesis may provide competitive solutions to realize nanomaterial generation by modest and cost-effective methods. Although this approach still has room for improvement by 
controlling various factors influencing the performances of the prepared materials, it is meaningful to introduce a novel synthetic methodology beneficial to a wide range of materials science community.

\section{Methods}

Synthesis of functional nanocrystals. The starting materials and solvents used to prepare precursor solution for the functional nanocrystals is as given below:

CdS. $1 \mathrm{M}$ of Cadmium Acetate dihydrate $\left(\mathrm{Cd}\left(\mathrm{C}_{2} \mathrm{H}_{3} \mathrm{O}_{2}\right)_{2} \cdot 2 \mathrm{H}_{2} \mathrm{O}\right.$, Aldrich) and thiourea $\left(\mathrm{CH}_{4} \mathrm{~N}_{2} \mathrm{~S}\right.$, Aldrich) were dissolved in tetraethylene glycol $\left(\left(\mathrm{HOCH}_{2} \mathrm{CH}_{2}\right)_{2} \mathrm{O}, 99 \%\right.$, Daejung Chemicals).

$\mathrm{ZnO} .1 \mathrm{M} \mathrm{Zn}\left(\mathrm{CH}_{3} \mathrm{COO}\right)_{2} \cdot 2 \mathrm{H}_{2} \mathrm{O}$ was dissolved in ethylene glycol $\left(\mathrm{HOCH}_{2} \mathrm{CH}_{2} \mathrm{OH}\right.$, Daejung Chemicals).

$\mathrm{TiO}_{2}$. 1M Titanium isopropoxide $\left(\mathrm{Ti}\left[\mathrm{OCH}\left(\mathrm{CH}_{3}\right)_{2}\right]_{4}\right.$, Junsei) was dissolved in tetraethylene glycol.

$\mathrm{LiFePO}$. Lithium acetate dihydrate $\left(\mathrm{CH}_{3} \mathrm{COOLi} \cdot 2 \mathrm{H}_{2} \mathrm{O}\right.$, GR, Junsei) and iron acetate ( $\mathrm{Fe}\left(\mathrm{CH}_{3} \mathrm{COO}\right)_{2}$, Aldrich) corresponding to $1 \mathrm{M} \mathrm{LiFePO}_{4}$ was dissolved in tetraethylene glycol. $1 \mathrm{M}$ Phosphoric acid $\left(\mathrm{H}_{3} \mathrm{PO}_{4}\right.$, Daejung Chemicals) was then stirred with the resulting solution until it became homogenous.

Ag. $0.01 \mathrm{M}$ of Silver nitrate $\left(\mathrm{AgNO}_{3}, 99+\%\right.$, Aldrich) was dissolved in $80 \mathrm{ml}$ tetraethylene glycol.

The flammable solution was ignited with a torch to induce a self-extinguishable combustion process. Highly crystalline nanoparticles were obtained.

Powder X-ray diffraction. The PXRD patterns were measured using Shimadzu X-ray Diffractometer with $\mathrm{Ni}$-filtered $\mathrm{Cu} \mathrm{K} \alpha$ radiation $(\lambda=1.5406 \AA)$ operating at $40 \mathrm{kV}$ and $30 \mathrm{~mA}$ in the scanning angle, $2 \theta$, range of $10-80^{\circ}$ in steps of $0.02^{\circ}$. The lattice parameters were obtained by the whole-pattern profile matching method using the FULLPROF ${ }^{52}$ program.

Electron microscopy (HR-TEM and FE-SEM) analyses. SEM images were obtained using S-4700 from HITACHI and TEM pictures were recorded using FEI Tecnai F20 at $200 \mathrm{kV}$.

Electrochemical characterization. For electrochemical measurements, the carboncoated $\mathrm{LiFePO}_{4}$ and pure $\mathrm{TiO}_{2}$ active materials were mixed with 25 and $30 \mathrm{wt} \%$ of conducting carbon respectively taking into account the $5 \mathrm{wt} \%$ carbon already present in the carbon content in the former sample and teflonized acetylene black (TAB) was used as binder. Usually, a loading of $3.5 \mathrm{mg} \mathrm{cm}^{-2}$ as the active material was used. The mixture was pressed onto a stainless steel mesh and vacuum dried at $120^{\circ} \mathrm{C}$ for 12 hours, thus forming the cathode. A 2032 coin type cell consisting of the cathode and lithium metal anode separated by a polymer membrane was fabricated in an $\mathrm{Ar}$ filled glove box and aged for 12 hours. The electrolyte employed was a 1:1 mixture of ethylene carbonate (EC) and dimethylcarbonate (DMC) containing $1 \mathrm{M} \mathrm{LiPF}_{6}$. Galvanostatic tests were carried out at room temperature using BTS 2004H (Nagano, Japan).

UV-visible spectrophotometer test. For measuring optical properties of CdS and $\mathrm{ZnO}$ nanocrystals, optical absorption studies were performed in the wavelength of 300-800 nm using UV-visible spectrophotometer (Cary 100, Varian, Mulgrave, Australia) at room temperature.

1. Burda, C., Chen, X., Narayanan, R. \& El-Sayed, M. A. Chemistry and properties of nanocrystals of different shapes. Chem. Rev. 105, 1025-1102 (2005).

2. Rao, C. N. R., Kulkarni, G. U., Thomas, P. J. \& Edwards, P. P. Size-dependent chemistry: Properties of nanocrystals. Chem. Eur. J. 8, 28-35 (2002).

3. Ma, R. \& Sasaki, T. Nanosheets of oxides and hydroxides: ultimate 2D chargebearing functional crystallites. Adv. Mater. 22, 5082-5104 (2010).

4. Alivisatos, P. The use of nanocrystals in biological detection. Nat. Biotechnol 22, 47-52 (2004).

5. Kim, J. \& Manthiram, A. A manganese oxyiodide cathode for rechargeable lithium batteries. Nature 390, 265-267 (1997).

6. Arico, A. S., Bruce, P., Scrosati, B., Tarascon, J.-M. \& van Schalkwijk, W. Nanostructured materials for advanced energy conversion and storage devices. Nat. Mater. 4, 366-377 (2005).

7. Kamat, P. V. Quantum dot solar cells. Semiconductor nanocrystals as light harvesters. J. Phys. Chem. C 112, 18737-18753 (2008).

8. Law, M., Greene, L. E., Johnson, J. C., Saykally, R. \& Yang, P. Nanowire dyesensitized solar cells. Nat. Mater. 4, 455-459 (2005).

9. Chan, C. K. et al. High-performance lithium battery anodes using silicon nanowires. Nat. Nanotechnol 3, 31-35 (2008).

10. Simon, P. \& Gogotsi, Y. Materials for electrochemical capacitors. Nat. Mater. 7, 845-854 (2008).
11. Huang, H., Yin, S. C. \& Nazar, L. F. Approaching theoretical capacity of $\mathrm{LiFePO}_{4}$ at room temperature at high rates. Electrochem. Solid-State Lett. 4, A170-A172 (2001).

12. Chung, S. Y., Bloking, J. T. \& Chiang, Y. M. Electronically conductive phosphoolivines as lithium storage electrodes. Nat. Mater. 1, 123-128 (2002).

13. Long, J. W., Dunn, B., Rolison, D. R. \& White, H. S. Three-dimensional battery architectures. Chem. Rev. 104, 4463-4492 (2004).

14. Yang, H., Wu, X. L., Cao, M. H. \& Guo, Y. G. Solvothermal synthesis of $\mathrm{LiFePO}_{4}$ hierarchically dumbbell-like microstructures by nanoplate self-assembly and their application as a cathode material in lithium-ion batteries. J. Phys. Chem. C. 113, 3345-3351 (2009)

15. Gutsch, A., Muhlenweg, H. \& Kramer, M. Tailor-made nanoparticles via gasphase synthesis. Small 1, 30-46 (2005).

16. Hernandez, R. J., Checot, F., Gnanou, Y. \& Lecommandoux, S. Toward 'smart' nano-objects by self-assembly of block copolymers in solution. Prog. Polym. Sci. 30, 691-724 (2005)

17. Yamada, A., Chung, S. C. \& Hinokuma, K. Optimized $\mathrm{LiFePO}_{4}$ for lithium battery cathodes. J. Electrochem. Soc. 148, A224-A229 (2001).

18. Demazeau, G. Solvothermal processes: a route to the stabilization of new materials. J. Mater. Chem. 9, 15-18 (1999).

19. Therese, G. H. A. \& Kamath, P. V. Electrochemical synthesis of metal oxides and hydroxides. Chem. Mater. 12, 1195-1204 (2000).

20. Yang, S., Zavalij, P. Y. \& Whittingham, M. S. Hydrothermal synthesis of lithium iron phosphate cathodes. Electrochem. Commun. 3, 505-508 (2001).

21. Arnold, G. et al. Fine-particle lithium iron phosphate $\mathrm{LiFePO}_{4}$ synthesized by a new low-cost aqueous precipitation. J. Power Sources 119-121, 247-251 (2003).

22. Lukac, J. et al. Characterization of $\mathrm{Zr}$-doped $\mathrm{TiO}_{2}$ prepared by homogenous coprecipitation without high-temperature treatment. J. Mater. Sci. 42, 9421-9428 (2007).

23. Niederberger, M. Nonaqueous sol-gel routes to metal oxide nanoparticles. Acc. Chem. Res. 40, 793-800 (2007).

24. Sun, X. M. \& Li, Y. D. Cylindrical silver nanowires: preparation, structure, and optical properties. Adv. Mater. 17, 2626-2630 (2005).

25. Banger, K. K. et al. Low-temperature, high-performance solution-processed metal oxide thin-film transistors formed by a 'sol-gel on chip' process. Nat. Mater. 10, $45-50$ (2011).

26. McCormick, P. G., Tsuzuki, T., Robinson, J. S. \& Ding, J. Nanopowders synthesized by mechanochemical processing. Adv. Mater. 13, 1008-1010 (2001).

27. Hahn, H. Gas phase synthesis of nanocrystalline materials. Nanostruct. Mater. 9, 3-12 (1997).

28. Song, Y., Hormes, J. \& Kumar, C. S. S. R. Microfluidic synthesis of nanomaterials. Small 4, 698-711 (2008)

29. Bilecka, I. \& Niederberger, M. Microwave chemistry for inorganic nanomaterials synthesis. Nanoscale, 2, 1358-1374 (2010).

30. Aruna, S. T. \& Mukasyan, A. Combustion synthesis and nanomaterials. Curr. Opin. Solid State Mater. Sci. 12, 44-50 (2008).

31. Figlarz, M., Fievet, F. \& Lagier, J. P. Process for the reduction of metallic compounds by polyols, and metallic powders obtained by this process. $U$. S. Patent 1985/4539041.

32. Feldmann, C. Polyol mediated synthesis of nanoscale functional materials. $A d v$ Funct. Mater. 13, 101-107 (2003).

33. Laoutid, F., Bonnaud, L., Alexandre, M., Lopez-Cuesta, J. M. \& Dubois, Ph. New prospects in flame retardant polymer materials: From fundamentals to nanocomposites. Mat. Sci. Eng., R 63, 100-125 (2009).

34. Padhi, A. K., Nanjundaswamy, K. S. \& Goodenough, J. B. Phospho-olivines as positive-electrode materials for rechargeable lithium batteries. J. Electrochem. Soc. 144, 1188-1194 (1997).

35. Chen, X. \& Mao, S. S. Titanium dioxide nanomaterials: Synthesis, properties, modification, and application. Chem. Rev. 107, 2891-2959 (2007).

36. Kim, M. G., Kanatzidis, M. G., Facchetti, A. \& Marks, T. J. Low-temperature fabrication of high-performance metal oxide thin-film electronics via combustion processing. Nat. Mater. 10, 382-388 (2011).

37. Murray, C. B., Norris, D. J. \& Bawendi, M. G. Synthesis and characterization of nearly monodisperse $\mathrm{CdE}(\mathrm{E}=\mathrm{S}, \mathrm{Se}, \mathrm{Te})$ semiconductor nanocrystallites. J. Am. Chem. Soc. 115, 8706-8715 (1993).

38. Zhai, C. et al. One-pot synthesis of biocompatible CdSe/CdS quantum dots and their applications as fluorescent biological labels. Nanoscale Res. Lett. 6, 31-35 (2011).

39. Delacourt, C., Poizot, P., Levasseur, S. \& Masquelier, C. Size effects on carbon-free $\mathrm{LiFePO}_{4}$ powders: The key to superior energy density. Electrochem. Solid-State Lett. 9, A352-A355 (2006).

40. Cho, S., Jang, J. W., Lee, J. S. \& Lee, K. H. Carbon-doped ZnO nanostructures synthesized using vitamin C for visible light photocatalysis. Cryst. Eng. Comm. 12, 3929-3935 (2010).

41. Ye, A., Fan, W., Zhang, Q., Deng, W. \& Wang, Y. CdS-graphene and CdS-CNT nanocomposites as visible-light photocatalysts for hydrogen evolution and organic dye degradation. Catal. Sci. Technol 2, 969-978 (2012).

42. Ravet, N. et al. Electroactivity of natural and synthetic triphylite. J. Power Sources 97-98, 503-507 (2001)

43. Rycenga, M. et al. Controlling the synthesis and assembly of silver nanostructures for plasmonic applications. Chem. Rev. 111, 3669-3712 (2011).

44. Schoen, D. T. et al. High speed water sterilization using one-dimensional nanostructures. Nano Lett. 10, 3628-3632 (2010). 
45. Kim, D., Jeong, S. \& Moon, J. Synthesis of silver nanoparticles using the polyol process and the influence of precursor injection. Nanotechnology 17, 4019-4024 (2006).

46. Kim, D. H. \& Kim, J. Synthesis of $\mathrm{LiFePO}_{4}$ nanoparticles in polyol medium and their electrochemical properties. Electrochem. Solid-State Lett. 9, A439-A442 (2006).

47. Tripathi, R., Ramesh, T. N., Ellis, B. L. \& Nazar, L. F. Scalable synthesis of tavorite $\mathrm{LiFeSO}_{4} \mathrm{~F}$ and $\mathrm{NaFeSO}_{4} \mathrm{~F}$ cathode materials. Angew. Chem. Int. Ed. 49, 8738-8742 (2010).

48. Chakroune, N., Viau, G., Ricolleau, C., Vincent, F. F. \& Fiévet, F. Cobalt-based anisotropic particles prepared by the polyol process. J. Mater. Chem. 13, 312-318 (2003).

49. Chiang, Y. M., Gozdz, A. S. \& Payne, M. W. Nanoscale ion storage materials. U.S. Patent No. US 2007/0031732 A1.

50. Saidi, M. Y. \& Huang, H. Synthesis of metal phosphates. US Patent No. US 2006/ 7060238 B2.

51. Adamson, G., Barker, J. \& Saidi, M. Y. Secondary battery electrode active materials and methods for making the same. U.S. Patent No. US 2006/7008726 B2.

52. Carvajal, J. R. Satellite Meeting on Powder Diffraction of the XV Congress of the IUCr, Toulouse, France, pp.127 (1990).

\section{Acknowledgments}

This research was supported by World Class University (WCU) program through the Korea Science and Engineering Foundation funded by the Ministry of Education, Science and
Technology (R32-20074). We are grateful to Dr. Eunjoung Kim and Ms. Insun Yoo for their help with some experimental works. We are thankful to Mr. Junhee Han and Mr.

Seung-Wook Shin for contributing to TEM analysis and optical measurements respectively.

\section{Author contributions}

J.K. proposed the concept. The authors V.M. and J.G. contributed equally to this work. V.M and J.G. analyzed and wrote the manuscript and V.M. contributed to a major portion of the writing. J.G. prepared a major portion of the figures. J.S., S.B. and J.K. performed experiments and V.M., J.G. and J.L. contributed to technical discussions of results. D.A. analyzed Powder X-ray diffraction data and S.J.S. contributed to thermodynamic calculations and analysis. J.K. and H.Y. developed the synthesis scheme figure. All authors reviewed the paper and V.M., J.K. and J.G. performed the revisions.

\section{Additional information}

Supplementary information accompanies this paper at http://www.nature.com/ scientificreports

Competing financial interests: The authors declare no competing financial interests. License: This work is licensed under a Creative Commons Attribution-NonCommercial-NoDerivs 3.0 Unported License. To view a copy of this license, visit http://creativecommons.org/licenses/by-nc-nd/3.0/

How to cite this article: Gim, J. et al. Pyro-Synthesis of Functional Nanocrystals. Sci. Rep. 2, 946; DOI:10.1038/srep00946 (2012). 